\title{
Bronchoalveolar lavage fluid review in acute promyelocytic leukemia differentiation syndrome
}

\author{
Robert P. Seifert ${ }^{1}$ (D) $\cdot$ Scott Gregory ${ }^{1}$ \\ Received: 26 October 2020 / Accepted: 24 November 2020 / Published online: 17 January 2021 \\ (C) The Author(s), under exclusive licence to Springer-Verlag GmbH, DE part of Springer Nature 2021
}

\begin{abstract}
The patient was a 62-year-old Caucasian man with blood smear and flow cytometry concerning for acute promyelocytic leukemia with FISH ultimately confirming PML-RARA translocation. He had a 30-year history of employment at a nuclear power plant. He presented with diffuse intravascular coagulation, hyperleukocytosis, and quickly developed acute respiratory distress syndrome. On day four of ATRA + Hydrea, a bronchoalveolar lavage was performed and was non-bloody. On microscopic fluid review, abnormal immature cells with bilobed nuclear contours were identified, similar in morphology to those seen on the diagnostic blood smear review, amidst background alveolar-type macrophages. Subsequent flow cytometric analysis showed these cells to be abnormal promyelocytes; however, they differed from the blood flow cytometry study performed prior to initiation of ATRA by showing maturational immunophenotypic changes. While the leukemic promyelocytes on bronchoalveolar lavage were morphologically immature, these immunophenotypic changes somewhat recapitulated those seen in normal granulocyte maturation and were thus suggestive of so-called differentiation syndrome. Unfortunately, the patient passed away during induction chemotherapy due to complications from diffuse intravascular coagulation and differentiation syndrome. Important pathobiological information can be gathered from fluid review and concomitant flow cytometric analysis.
\end{abstract}

Keywords APL $\cdot$ Differentiation syndrome $\cdot$ ATRA $\cdot$ Flow cytometry $\cdot$ Fluid review $\cdot$ Acute promyelocytic leukemia

\section{Introduction}

Acute promyelocytic leukemia (APL) with PML-RARA is driven by a translocation, $\mathrm{t}(15 ; 17)$, pairing the $P M L$ gene of chromosome 15 with the retinoic acid receptor-a gene of chromosome 17. This arrests granulocyte development at the promyelocyte stage leading to an accumulation of leukemic promyelocytes [1]. Therapy consists of high doses of all-trans retinoic acid (ATRA) in addition to anthracycline chemotherapy and arsenic trioxide. Complete remission is achieved with the above modalities in greater than $90 \%$ of patients and the 5 year overall survival rate is $80 \%$ [2]. Treatment success can be attributed to ATRA's mechanism of action which relieves the blockage induced by the translocation, allowing the

Robert P. Seifert

rseifert@ufl.edu

1 Department of Pathology, Immunology, and Laboratory Medicine, University of Florida, 1600 SW Archer Rd, Gainesville, FL 32608, USA promyelocytes to continue differentiating into mature granulocytes [3]. However, induction mortality due to socalled differentiation syndrome remains an issue [4].

Differentiation syndrome presents with acute respiratory distress, weight gain, unexplained fever, interstitial pulmonary infiltrates, pleural/pericardial effusions, hypotension, and acute renal failure. It is reported to occur, during induction, in between 2.5 and $31 \%$ of APL patients treated with ATRA and is associated with $15 \%$ of induction deaths [4]. It is a diagnostic challenge that is incumbent upon identification of the aforementioned signs/symptoms which may be confounded by existing co-morbidities in a given patient such as congestive heart failure. No specific and reliable lab test exists for early identification. Pathogenesis of differentiation syndrome is not fully understood but involves hyperactivation of a number of inflammatory mechanisms. Some studies have proposed CCL2 and CXCL8 excess in differentiation syndrome drive the differentiating promyelocytic cells to migrate towards alveoli [4]. We present a patient with APL and differentiation syndrome in which promyelocytes were identified on bronchoalveolar lavage that demonstrated progressive maturation by flow cytometric analysis. 


\section{Clinical history}

The patient was a 62-year-old Caucasian man who presented to the emergency department with a 3-week history of cough, diarrhea, decreased appetite, weight loss, and epistaxis. His past medical history included type 2 diabetes mellitus, hypertension, hyperlipidemia, and Factor $\mathrm{V}$ Leiden mutation for which he took apixaban. He had a history of smoking and a 30 -year history of working at a nuclear power plant. His initial CBC with manual differential showed WBC 127,000/uL, Hgb $7.2 \mathrm{~g} / \mathrm{dL}$, and platelets $7000 / \mathrm{uL}$ with $90 \%$ "blasts." LDH was 908 IU/L. Apixaban was held after thrombocytopenia was identified and the patient was later started on unfractionated heparin. Further coagulation studies showed that he had an elevated D-Dimer of greater than $20 \mu \mathrm{g} / \mathrm{mL}$ (reference range $<0.5 \mu \mathrm{g} / \mathrm{mL}$ ) and a decreased fibrinogen $41 \mathrm{mg} / \mathrm{dL}$ (reference range 173-454 mg/dL). Prothrombin time was elevated, $20 \mathrm{~s}$ (reference range 9.1 to $13.5 \mathrm{~s}$ ), while aPTT was within normal limits $27 \mathrm{~s}$ (reference range $25-38 \mathrm{~s}$ ). SARS-COV-2 nucleic acid testing on nasopharyngeal swab was negative.

On blood smear review, the "blasts" had bilobed nuclear contours with occasional Auer rods (Fig. 1A). Blood flow cytometric analysis detected a CD34(virtually negative), CD117(dim+), and CD123(+) population of abnormal promyelocytes without HLA-DR or CD15 expression, immunophenotypically compatible with acute promyelocytic leukemia. Myeloperoxidase cytochemistry was strongly positive in the leukemic promyelocytes. All-trans retinoic acid (ATRA) therapy was initiated in addition to Hydrea and Prednisone. The definitive diagnosis of acute promyelocytic leukemia with PMLRARA was confirmed by FISH testing $16 \mathrm{~h}$ later. FLT3-ITD was detected by PCR with no other relevant pathogenic variants detected by next generation sequencing.

Bilateral ischemic changes were noted in the lower extremities, concerning for diffuse intravascular coagulation. AP chest X-Ray noted bilateral airspace opacities on day four of
ATRA and this was confirmed by non-contrast chest computed tomography scan. This was concerning for differentiation syndrome. A bronchoalveolar lavage was performed and was non-bloody with $5 \%$ mature neutrophils, $22 \%$ lymphocytes, $6 \%$ monocytes, and $67 \%$ blast-like cells. The blast-like cells had bilobed nuclear contours, similar to the promyelocytes identified on the patient's diagnostic blood smear (Fig. 1). Flow cytometric analysis detected a population of abnormal promyelocytes with dim CD117 and aberrant CD123 expression. Unlike the promyelocytes seen on the blood specimen, this population showed maturational expression of $\mathrm{CD} 11 \mathrm{~b}$ (Fig. 2). Unfortunately, the patient passed away during induction chemotherapy due to complications from diffuse intravascular coagulation and differentiation syndrome.

\section{Materials and methods}

The BAL specimen was received fresh for flow cytometry. Erythrocytes were lysed by incubating with lysing solution (15 mM NH4Cl, $1.0 \mathrm{mM} \mathrm{KHCO3,} 10 \mathrm{mM}$ EDTA, and $\mathrm{pH}$ 7.4) for $10 \mathrm{~min}$ at room temperature. The cells were then resuspended in phosphate-buffered saline with $0.1 \% \mathrm{NaN} 3$ (PBS), and washed twice in PBS. The cell viability was confirmed by morphology and 7-Aminoactinomycin D testing.

A four-tube immunophenotyping panel for the evaluation of myeloid neoplasms was performed on both the blood and bronchoalveolar lavage specimens (Table 1). Fluorochromes used included fluorescein isothiocyanate (FITC), phycoerythrin (PE), peridinin chlorophyll protein-cyanine 5.5 (PerCP-Cy5.5), allophycocyanin (APC), allophycocyanin-cyanine tandem conjugate (APC-H7), and phycoerythrin-cyanine 7 (PE-Cy7). Cells $(200,000)$ were incubated with fluorophoreconjugated antibodies in $200 \mu \mathrm{L}$ of Hanks balanced salt solution (Mediatech Cellgro, Herndon, VA, USA) with

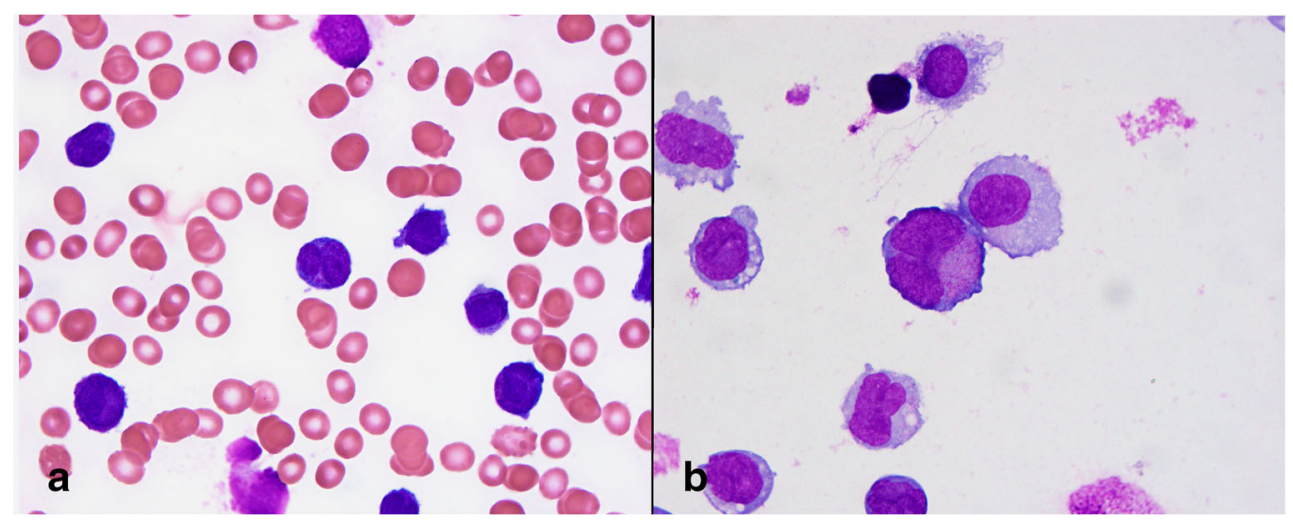

Fig. 1 a Blood, Wright-Giemsa stain, 1000x, frequent abnormal promyelocytes with bilobed nuclear contours and azurophilic granules. b Bronchoalveolar lavage cytospin preparation, Wright-Giemsa stain, 1000x, occasional immature cells some of which have bilobed nuclear

contours and azurophilic granules. They are larger in size than the circulating promyelocytes seen at diagnosis, but this may be artifactual. Background alveolar macrophages are also noted 

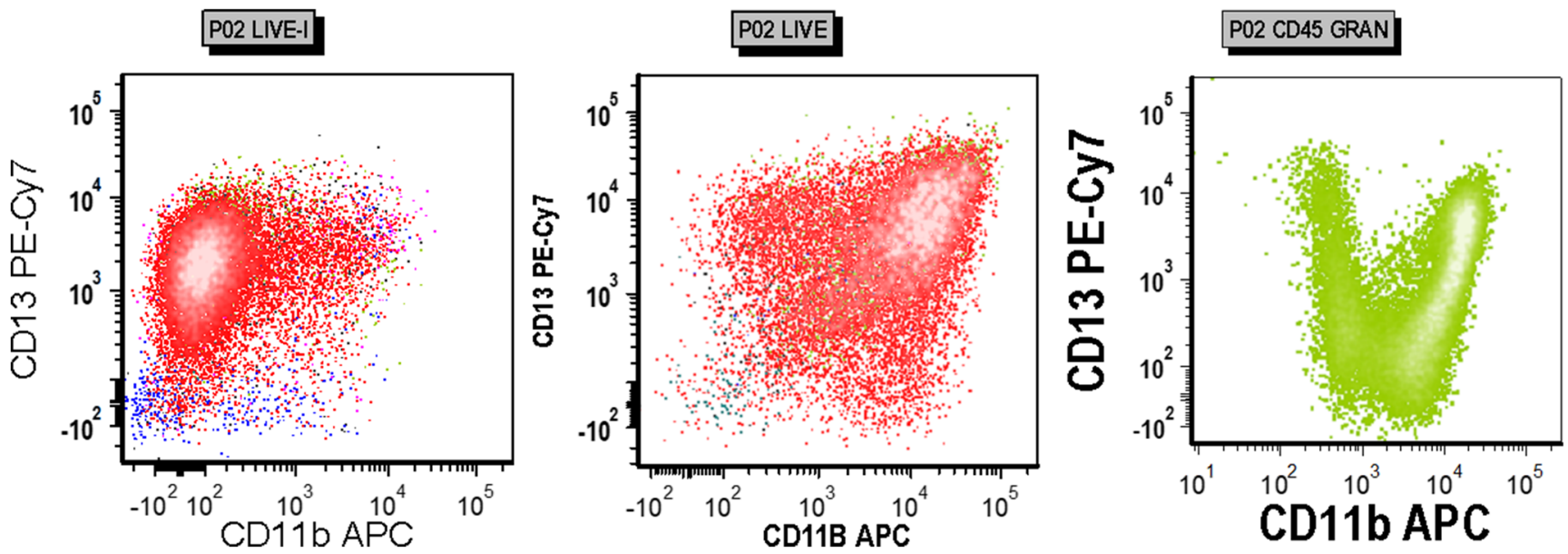

Fig. 2 Flow cytometry scatterplots with white shading by density. Based on side light scatter characteristics and CD45 expression, leukemic cells are colored red, granulocytes light green, and lymphocytes blue. Leukemic cells in the diagnostic blood specimen (left, gated on live events) showed bright CD13 expression with little to no expression of CD11b. The bronchoalveolar lavage specimen (middle, gated on live

5\% human $\mathrm{AB}$ serum (Bio-Whittaker, Walkersville, MD, USA) for $15 \mathrm{~min}$ on ice without light exposure. Subsequently, the cells were washed twice with PBS by centrifugation at $500 \times \mathrm{g}$ for $5 \mathrm{~min}$ and resuspended in a final volume of $250 \mu \mathrm{L}$ of PBS. Six-color flow cytometry was performed using a FACSCanto II flow cytometer (Becton Dickinson [BD], San Jose, CA, USA) equipped with a 488-nm argon laser, a 635-nm diode laser, and FACSDiva software (BD). Daily calibration of the instrument was performed using standardized CaliBRITE Beads (BD) with FACSDiva Software (BD). On instrument fluorescence compensation was performed using settings from compensation matrices that were confirmed daily using blood mononuclear cells. A minimum of 20,000 events were acquired in each tube. Doublets were eliminated by gating on single cells based on forward scatter area and width. Live cells and mononuclear cells were then gated based on forward and side light scatter characteristics. Different cell populations were identified based on side scatter characteristics and CD45 expression with granulocytes colored in light green, lymphocytes in blue, monocytes in pink, leukemic cells in red, and CD45(-) cells in teal. events) showed a similar proportion of CD13 expression with progressive CD11B expression. This recapitulated normal granulocyte maturation by CD13 and CD11b expression in the bone marrow as demonstrated by an unrelated normal bone marrow specimen (right, gated on granulocytes)

\section{Results}

The patient's blood smear contained frequent (90\%) abnormal bilobed promyelocytes with azurophilic granules. Only rare cells contained Auer rods (Fig. 1a). Cytochemistry for myeloperoxidase showed classic "spiking." Flow cytometric analysis showed an immunophenotype consistent with acute promyelocytic leukemia including dim CD117 expression with near absence of CD34. CD15 and CD11b were negative while HLA-DR was variable and CD123 was aberrantly positive. The cytospin preparation prepared from the bronchoalveolar lavage demonstrated morphologically similar promyelocytes (Fig. 1b) albeit larger in size, although this was likely an artifact of preparation. However, flow cytometric analysis demonstrated maturational changes in CD11b expression (Fig. 2, middle). This pattern somewhat recapitulates what is seen in normal granulocyte maturation (Fig. 2, right).

\section{Discussion}

Differentiation syndrome in acute promyelocytic leukemia is not a well-understood process and likely involves

Table 1 Six color flow cytometry panel (antibody, vendor, clone)

\begin{tabular}{|c|c|c|c|c|c|c|}
\hline Flourochromes & FITC & $\mathrm{PE}$ & PerCP-Сy5.5 & $\mathrm{APC}$ & APC-H7 & PE-Cy7 \\
\hline Tube 1 & CD38, Coulter, T16 & $\mathrm{CD} 117, \mathrm{BD}, 104 \mathrm{D} 2$ & $\mathrm{CD} 34, \mathrm{BD}, 8 \mathrm{G} 12$ & CD71, Coulter, L01.1 & $\mathrm{CD} 45, \mathrm{BD}, 2 \mathrm{D} 1$ & CD56, Coulter, N901 \\
\hline Tube 2 & CD16, BD, NKP15 & CD7, BD, M-T701 & $\mathrm{CD} 34, \mathrm{BD}, 8 \mathrm{G} 12$ & $\mathrm{CD} 11 \mathrm{~b}, \mathrm{BD}, \mathrm{D} 12$ & $\mathrm{CD} 45, \mathrm{BD}, 2 \mathrm{D} 1$ & CD13, BD, L138 \\
\hline Tube 3 & CD15, Coulter, H0H5 & CD33, BD, P67.6 & $\mathrm{CD} 123, \mathrm{BD}, 7 \mathrm{G} 3$ & HLA-DR, BD, L243 & $\mathrm{CD} 45, \mathrm{BD}, 2 \mathrm{D} 1$ & $\begin{array}{l}\text { CD117, } \\
\quad \text { Coulter, 104D2D1 }\end{array}$ \\
\hline Tube 4 & CD64, Coulter, 22 & CD41, Coulter, P2 & HLA-DR, BD, L243 & CD117, BD 104D2 & $\mathrm{CD} 45, \mathrm{BD}, 2 \mathrm{D} 1$ & CD14, BD, M5E2 \\
\hline
\end{tabular}


a number of mechanisms triggered by ATRA administration, some of which enhance pulmonary homing of leukemic cells. In addition to damage caused by the release of numerous inflammatory cytokines, promyelocytes themselves undergo changes that alter cytokine release and adhesion-leading to increased tissue infiltration and morbidity. These changes are felt to represent maturation of the leukemic promyelocytes due to ATRA's action on the blocked retinoic acid receptor. The patient's condition declined rapidly. While many factors may have played a role, the patient's history of Factor V Leiden, for which he was chronically anticoagulated prior to admission, may have contributed to his progressive diffuse intravascular coagulation.

Other authors have observed increased CD11b expression in APL promyelocytes during ATRA administration [4], as we have in this case, which can correlate with maturation patterns observed in normal granulocytic precursors [5]. In this case, immunophenotypic findings provided support to the clinical diagnosis of differentiation syndrome. Important pathobiological information can be gathered from fluid review and concomitant flow cytometric analysis if differentiation syndrome is a clinical consideration.

\section{Compliance with ethical standards}

Conflict of interest The authors have no relevant financial conflicts of interest to disclose.

\section{References}

1. de Thé H, Lavau C, Marchio A, Chomienne C, Degos L, Dejean A (1991) The PML-RAR $\alpha$ fusion mRNA generated by the $t(15 ; 17)$ translocation in acute promyelocytic leukemia encodes a functionally altered RAR. Cell 66:675-684

2. Wang Z-Y, Chen Z (2008) Acute promyelocytic leukemia: from highly fatal to highly curable. Blood 111:2505-2515

3. Castaigne S, Chomienne C, Daniel MT et al (1990) All-trans retinoic acid as a differentiation therapy for acute promyelocytic leukemia. I Clinical results

4. Luesink M, Pennings JL, Wissink WM et al (2009) Chemokine induction by all-trans retinoic acid and arsenic trioxide in acute promyelocytic leukemia: triggering the differentiation syndrome. Blood, The Journal of the American Society of Hematology 114:5512-5521

5. Van Lochem E, Van der Velden V, Wind H et al (2004) Immunophenotypic differentiation patterns of normal hematopoiesis in human bone marrow: reference patterns for age-related changes and disease-induced shifts. Cytometry Part B: Clinical Cytometry: The Journal of the International Society for Analytical Cytology 60:1-13

Publisher's note Springer Nature remains neutral with regard to jurisdictional claims in published maps and institutional affiliations. 